\title{
Analysis of the impact of surface heat transfer on a new modification of the Angstroem's method
}

\author{
by C. Bussi, A. Muscio, M.A. Corticelli
}

DIMeC - Dipartimento di Ingegneria Meccanica e Civile, Università di Modena e Reggio Emilia, Modena, Italia

\begin{abstract}
A new modification of the Angstroem's method for thermal diffusivity measurement has been developed. This relies on the propagation of harmonic thermal waves with mean value equal to the ambient temperature. The diffusivity is evaluated by relatively simple processing of temperature data, acquired by infrared thermography.

The evaluation is based on a mathematical model, in which the heat transfer coefficient at the specimen surface is assumed to be constant. This work is aimed at verifying that assumption. In particular, the effects of natural convection in air are investigated theoretically by numerical simulation. A strategy to improve the test procedure is finally outlined.
\end{abstract}

\section{Introduction}

A new technique for measurement of the thermal diffusivity has been developed, in collaboration between the DIMeC and the CNR-ITC Padova Section. The technique is a modification of the well-known Angstroem's method, which relies on the steady-periodic propagation of thermal waves along a specimen to estimate the diffusivity of the tested material [1]. The novelty of the proposed modification is that harmonic thermal waves with mean value equal to the ambient temperature are induced in the specimen by means of a purposely developed source, based on the Peltier effect. This allows evaluating the diffusivity by relatively simple processing of surface-temperature data, acquired by infrared thermography.

A cross comparison of the technique with other measurement methods gave promising results, showing the effectiveness of the approach to produce the thermal waves [2-6]. Accuracy and precision comparable with the standard flash method [7] were obtained.

Further improvements seem to be possible, with especial regard to controlling the test conditions. In fact, the propagation of the thermal waves is affected by heat transfer at the surface of the specimen. The coefficient of heat transfer with the test environment, which includes the superposed effects of thermal radiation and air convection, is generally unknown, but its estimate is made unnecessary by the procedure adopted to evaluate the diffusivity. In the mathematical model on which the evaluation procedure is based, however, a homogeneous and constant value is assumed for the heat transfer coefficient. This is not so easy to accept, since strong buoyancy effects can be unset in the air around the specimen by the propagation of the thermal waves. Therefore, the assumption is verified in this work, investigating either radiation or convection. Particular attention is paid to the surface pattern of the convection coefficient, which is theoretically estimated along the thermal cycles by numerical simulation. A strategy to limit the start of buoyancy effects is finally outlined. 


\section{Mathematical formulation}

The Angstroem's method is briefly described in the following for sake of completeness. A more detailed explanation is reported in [1-5].

Angstroem tested a long bar with small cross-section. One end of the bar was subjected to a periodic change of temperature, being alternately heated by a current of steam and cooled by a current of cold water, for equal time intervals. The bar was also allowed to exchange heat with an ambient at constant temperature. Assuming the temperature homogenous over the cross-section, the governing equation is:

$$
\frac{\partial T}{\partial t}=\alpha_{m} \frac{\partial^{2} T}{\partial x^{2}}-\frac{2 h P}{\rho_{m} c_{m} S}\left(T-T_{a}\right)
$$

where $x$ is the coordinate along the bar $(\mathrm{m}), t$ is the time $(\mathrm{s}), T$ is the temperature $(\mathrm{K})$, $h$ is the heat transfer coefficient at the bar surface $\left(\mathrm{W} \cdot \mathrm{m}^{-1} \cdot \mathrm{K}^{-1}\right), P$ and $S$ are the perimeter $(\mathrm{m})$ and the area $\left(\mathrm{m}^{2}\right)$ of the bar cross-section, $\alpha_{m}, \rho_{m}$ and $c_{m}$ are the thermal diffusivity $\left(\mathrm{m}^{2} \cdot \mathrm{s}\right)$, the density $\left(\mathrm{kg} \cdot \mathrm{m}^{-3}\right)$ and the specific heat $\left(\mathrm{J} \cdot \mathrm{kg}^{-1} \cdot \mathrm{K}^{-1}\right)$ of the tested material, $T_{a}$ is the ambient temperature.

In general, the boundary condition at the bar end periodically heated and cooled can be expressed by a Fourier's series. In connection with this, a steadyperiodic solution of Eq. (1), independent of the initial condition, always exists, but it is relatively complex and unpractical to be used for the estimate of the diffusivity. The solution is greatly simplified, however, if the boundary condition at $x=0$ is a perfectly harmonic oscillation of temperature about the ambient value:

$$
T(x=0, t)=T_{a}+\Delta T_{0} \sin \left(2 \pi t / t_{0}-\varphi_{0}\right)
$$

where $t_{0}, \Delta T_{0}$ and $\varphi_{0}$ are, respectively, the time cycle $(\mathrm{s})$, the amplitude $(\mathrm{K})$, and the initial phase (rad) of the temperature oscillation. The thermal cycle expressed by Eq. (2) can be obtained by a thermoelectric source based on the Peltier effect. The practical implementation of the source is described in $[4,6]$.

The temperature at the farther end is not affected by the alterations at $x=0$ if the bar is long enough (virtually semi-infinite) and one has the boundary condition:

$$
T(x \rightarrow \infty, t)=T_{a}
$$

The steady-periodic temperature field yielded along the bar is [1]:

$$
T(x, t)=T_{a}+\Delta T_{0} e^{-C x / L} \sin \left(2 \pi t / t_{0}-B x / L-\varphi_{0}\right)
$$

Eq. (4) describes a harmonic thermal wave, exponentially decaying while it travels along the bar. The amplitude decay and the phase shift are proportional to the so-called thermal diffusion length, $L$, a characteristic parameter depending on the thermal diffusivity of the tested material, $\alpha_{m}$, and the period of the thermal input, $t_{0}$ :

$$
L=\sqrt{\alpha_{m} t_{0} / \pi}
$$

The effects of heat transfer with the ambient are included in two parameters, $B$ and $C$, respectively affecting the phase and the amplitude of the thermal waves:

$$
B=\sqrt{-\left(t_{0} / t_{e}\right) /(2 \pi)+\sqrt{1+\left(t_{0} / t_{e}\right)^{2} /(2 \pi)^{2}}} \quad C=\sqrt{\left(t_{0} / t_{e}\right) /(2 \pi)+\sqrt{1+\left(t_{0} / t_{e}\right)^{2} /(2 \pi)^{2}}}
$$

Both parameters depend on an external relaxation time $t_{e}$, defined as follows: 


$$
t_{e}=\frac{\rho_{m} c_{m} S}{2 h P}
$$

The diffusivity can be estimated from the thermal diffusion length, $L$, which can in turn be calculated from the phase shift, $\varphi_{x}$, or from the logarithmic amplitude decay, $\Delta T_{x}$. Two apparent values of the diffusivity, $\alpha_{m, B}$ and $\alpha_{m, C}$, are obtained:

$$
\alpha_{m, B}=\alpha_{m} \frac{1}{B^{2}}=\frac{\pi / t_{0}}{\left(\partial \varphi_{x} / \partial x\right)^{2}} \quad \alpha_{m, C}=\alpha_{m} \frac{1}{C^{2}}=\frac{\pi / t_{0}}{\left[\partial\left(\ln \Delta T_{x}\right) / \partial x\right]^{2}}
$$

The apparent values are related to the actual one by two unknown factors, $1 / B^{2}(>1)$ and $1 / C^{2}(<1)$, both depending on heat transfer at the specimen surface. The true diffusivity can nevertheless be calculated by combining $\alpha_{m, B}$ and $\alpha_{m, c}$ :

$$
\alpha_{m}=\sqrt{\alpha_{m, B} \alpha_{m, C}}
$$

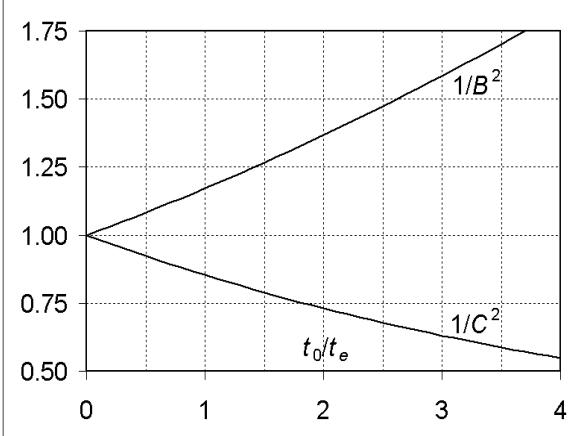

Fig. 1. Impact of heat exchange with the test ambient on the diffusivity estimate.

Heat exchange with the ambient may be made negligible, and both $1 / B^{2}$ and $1 / C^{2}$ made about equal to unity, by choosing a time cycle $t_{0}$ much smaller than $t_{e}$ (Fig. 1). This would yield values of apparent diffusivity equal to the actual one. In practice, the thermal inertia of the thermoelectric source, combined with the effects of convection and thermal radiation, imposes relatively large values of the ratio $t_{0} / t_{e}$. Therefore, Eq. (11) must generally be used. On the other hand, Eq. (11) has been obtain under the hypothesis of homogeneous and constant value of the heat transfer coefficient, $h$. The correctness of that hypothesis is verified in the following. In particular, thermal radiation and convection with air, whose superposition yields the total heat transfer between specimen an test ambient, are separately investigated.

\section{Impact of heat transfer with the test ambient by thermal radiation}

In the proposed measurement technique, the specimen is placed in a test ambient bounded by solid walls, as sketched in Fig. 2 . The wall temperature can be easily maintained homogenous and equal to the ambient temperature, $T_{a}$.

Air is transparent to thermal radiation. Therefore, the (plane) surfaces of the specimen exchange heat by radiation only with the walls of the test ambient. If the walls are coated black, reflection problems can be avoided and, above all, the rate of radiation heat transfer per unit surface can be evaluated by the relationship:

$$
q_{r}^{\prime \prime}=\varepsilon(T) \sigma_{0}\left(T^{4}-T_{a}^{4}\right) \cong h_{r}\left(T-T_{a}\right)
$$


where $\varepsilon$ is the surface emissivity of the specimen, $\sigma_{0}$ is the Stefan-Boltzmann constant (equal to $5.670 \times 10^{-8} \mathrm{~W} \cdot \mathrm{m}^{-2} \mathrm{~K}^{-4}$ ), $T$ is the local (surface) temperature of the specimen (K). The dependence on $T$ and $T_{a}$ has been made linear in Eq. (12) by introducing a heat transfer coefficient by radiation, $h_{r}$, defined as follows:

$$
h_{r}=\varepsilon(T) \sigma_{0}\left(T^{2}+T_{a}^{2}\right)\left(T+T_{a}\right)
$$

In principle, $h_{r}$ depends on $T$ explicitly and through the temperature dependence of $\varepsilon$. The latter can be neglected, however, if the amplitude of oscillation of $T$ about $T_{a}$ is small. This also allows equaling $h_{r}$ to its mean value, $h_{r, \text { mean: }}$ :

$$
h_{r} \cong h_{r, \text { mean }}=\varepsilon \sigma_{0} 4 T_{a}^{3}
$$

Fig. 3 shows that the relative deviation of $h_{r}$ from its mean value is always within $10 \%$ for $T_{a}$ above $300 \mathrm{~K}$ and $\Delta T_{0}$ below $20 \mathrm{~K}$ - conditions typically verified in the proposed measurement technique. Moreover, the deviation is positive along half cycle, negative in the other half, and its average value is about null. This suggests that fluctuations of $h_{r}$ are likely to have small or negligible impact on the phase shift and the amplitude decay of the thermal waves. Overall, it seems reasonable to assume homogeneous and constant the value of the radiation heat transfer coefficient and made it equal to its mean value, estimated by Eq. (14).

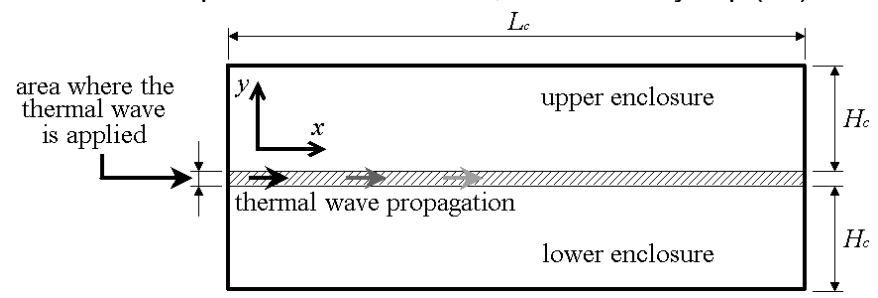

Fig. 2. Test ambient and bar specimen (longitudinal section).

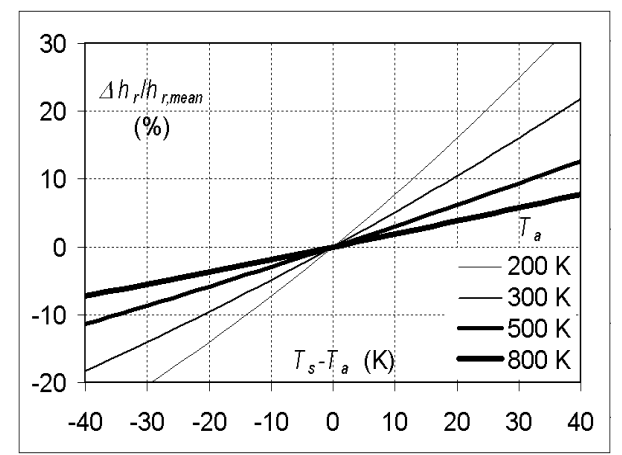

Fig. 3. $\Delta h_{r} / h_{r, \text { mean }}=\left(h_{r}-h_{r, \text { mean }}\right) / h_{r, \text { mean }}$, vs. $\left(T_{s}-T_{a}\right)$, for different values of $T_{a}$.

\section{Impact of convection in air within the test ambient}

The propagation of the thermal waves promotes buoyancy effects in the air around the specimen. As a result, heat exchange by convection, superposed to heat exchange by radiation, occurs between the specimen and the test ambient. The heat transfer coefficient $h$ in Eq. (1) can thus be calculated as the sum of the radiation component $h_{r}$, defined by Eq. (13), and a component due to natural convection, $h_{c}$. 
Differently from $h_{r}$, however, $h_{c}$ cannot be easily assumed to be homogenous and constant, since it is affected in an unpredictable manner by the unsteady distribution of temperature along the specimen. Moreover, the local value of $h_{c}$ cannot be calculated by analytical relationships. In fact, with transient natural convection, the only way to estimate theoretically the surface pattern of $h_{c}$ is by numerical simulation.

In this work, a parametric analysis has been carried out by numerical simulation, using a finite-volume based 'projection method', accurate to the 2 nd order in both space and time [8]. The physical problem that has been investigated is a rectangular cavity filled with air, representing each one of the two enclosures in which the test ambient is split by the immersed bar specimen (Fig. 2). The time-evolution of the temperature pattern expressed by Eq. (4) has been imposed at the boundary representing the specimen surface, whereas $T_{a}$ has been imposed elsewhere. A cavity with length $L_{c}$ much greater than the height $H_{c}$ has been considered, to assume the enclosures to be virtually infinite along the direction of wave propagation. Moreover, either the enclosures or the specimen have been assumed to be virtually infinite perpendicularly to the section in Fig 2 . This hypothesis is not completely realistic, but it makes the problem two-dimensional and computationally more affordable. Anyway, the simplification is adequate to the objective of this work, where the focus is more on the dynamics of natural convection than on its exact evaluation.

By dimensional analysis of the equations that govern natural convection in test ambient, it can be shown that, for a given material under testing, the distributions of velocity and temperature in air depend exclusively on the ratio between $H_{c}$ and the thermal diffusion length, $L$, and on two other dimensionless parameters, the Grashof number, Gr, and the Prandtl number, Pr. These are defined as:

$$
G r=\rho^{2} g \beta L^{3} \Delta T_{0} / \mu^{2} \quad \operatorname{Pr}=\mu c_{p} / \lambda
$$

where $g$ is the gravity acceleration (equal to $9.81 \mathrm{~m} \cdot \mathrm{s}^{-2}$ ), $\rho, \beta, \mu, c_{p}$, and $\lambda$ are, respectively, mass density $\left(\mathrm{kg} \cdot \mathrm{m}^{-3}\right)$, the thermal expansion coefficient $\left(\mathrm{K}^{-1}\right)$, the dynamic viscosity (Pa.s), the specific heat at constant pressure $\left(\mathrm{J} \cdot \mathrm{kg}^{-1} \cdot \mathrm{K}^{-1}\right)$ and the thermal conductivity $\left(\mathrm{W} \cdot \mathrm{m}^{-1} \cdot \mathrm{K}^{-1}\right)$ of the air in the test ambient (average values).

$\mathrm{Pr}$ is a physical property of air and it is about constant during an experiment. Therefore, the numerical analysis has been focused on $\mathrm{Gr}$ and the ratio $H_{c} / L$. Moreover, since the dependence on $H_{c} / L$ has been observed to be negligible for values of the ratio above 3 , a limit below which it is practically difficult to work, convection is actually controlled by the Grashof number alone.

Overall, the simulation demonstrated that, during the first half cycle, a vortex is produced in each enclosure, in the air near the region where the thermal waves are applied to the specimen. In the following half cycle, a new counter-rotating vortex is produced. The previous one is pushed away along the specimen and quickly vanishes. The streamlines show that the flow field in each enclosure is mirrored in the other one after half cycle, and it is already steady-periodic at the second cycle.

The dimensionless convection coefficient along the specimen surface is expressed by the Nusselt number, $\mathrm{Nu}$, defined as:

$$
N u=h_{c} L / \lambda
$$

The patterns of $\mathrm{Nu}$ calculated along the specimen are shown Fig. 4. On the left, different time instants of a cycle are considered. The patterns are clearly irregular in time and space, even if the peaks occur when heat transfer is about null $\left(T_{s} \cong T_{a}\right)$. On the right, different values of $\mathrm{Gr}$ are considered. $\mathrm{Nu}$ decreases with decreasing $G r$, approaching progressively the case of pure diffusion (i.e. with convection suppressed). The control of $\mathrm{Gr}$ is therefore desirable. 

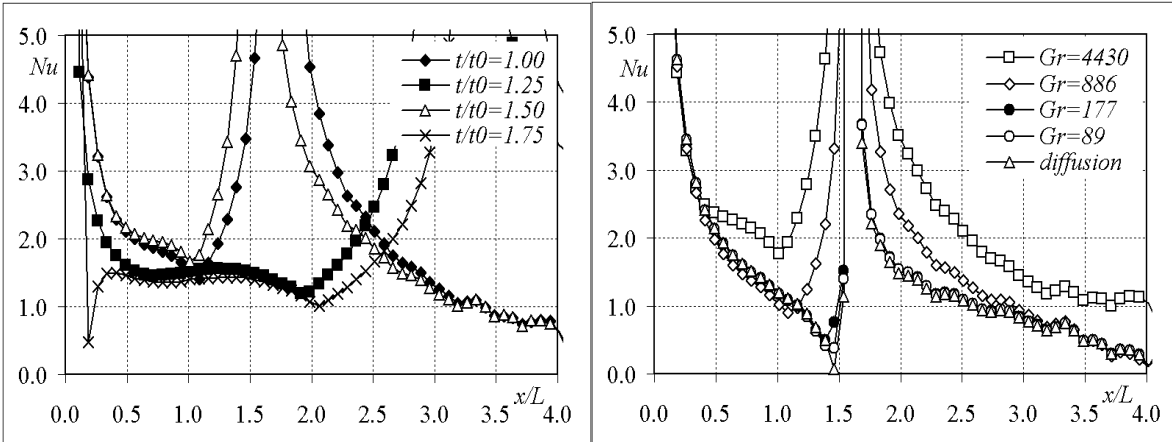

Fig. 4. $N u(x / L)$ vs. $t / t_{0}$ (left, $\left.G r=2658, H_{d} L=3\right)$ and vs. $G r$ (right, $\left.t / t_{0}=1.25, H_{d} L=3\right)$.

For given values of $\Delta T_{0}$ and $t_{0}$, imposed by the inertia of the thermoelectric source, the definition in Eq. (15) suggests that $\mathrm{Gr}$ can be reduced by increasing the viscosity, $\mu$, or decreasing the density, $\rho$, of the air. Both ones depend on $T_{a}$, but this is usually specified. Only $\rho$, however, is affected by pressure. In particular, $\rho$ decreases with decreasing pressure. Convection can thus be limited by working under vacuum. From the results summarized in Fig. 4 one obtains that a moderate vacuum (0.8-0.9 bar) is enough to make convection smaller than radiation $\left(h_{c} / h_{r}<0.2\right)$.

\section{Concluding remarks}

Convection in air, which is controlled by the Grashof number, was shown to produce an inhomogeneous and unsteady heat transfer coefficient. This is not desired in the Angstroem's method. Convection effects, however, can be reduced by working under moderate vacuum. This approach is currently being tested.

\section{REFERENCES}

[1] Carslaw, H.S., and Jaeger, J.C. Conduction of heat in solids, Oxford University Press, Oxford (1947).

[2] Bison, P.G., Muscio, A., and Grinzato, E. Thermal parameters estimation by heating and cooling and thermographic measurement, Proc. Thermosense XXI Conference, SPIE vol. 3700, pp. 402-408, Orlando, U.S.A. (1999).

[3] Marinetti, S., Bison, P.G., Grinzato, E., and Muscio, A. Thermal diffusivity measurement of stainless steel by periodic heating technique, Proc. $5^{\text {th }}$ AITA Int. Workshop, pp. 316-321, Venezia, Italia (1999).

[4] Muscio, A., and Grinzato, E. The lock-in heating-cooling method for the measurement of the thermal diffusivity of solid materials, Heat Transfer Engineering, vol. 23, n. 2, pp. 44-52 (2002).

[5] Bison, P.G., Marinetti, S., Mazzoldi, A., Grinzato, E., and Bressan, C. Crosscomparison of thermal diffusivity measurements by thermal methods, Infrared Physics and Technology, vol. 43, n. 3-5, pp. 127-132 (2002).

[6] Muscio, A., Bison, P.G., Marinetti, S., and Grinzato, E. Thermal diffusivity measurement in slabs using harmonic ad one-dimensional propagation of thermal waves, Int. J. Thermal Sciences, vol. 43, n. 5, pp. 453-563 (2004).

[7] ASTM, Standard test method for thermal diffusivities of solids by the flash method, Designation: E 1461-92 (1992).

[8] Barozzi, G.S., and Corticelli, M.A. Natural convection in cavities containing internal sources, Heat and Mass Transfer, vol. 36, pp. 473-480 (2000). 